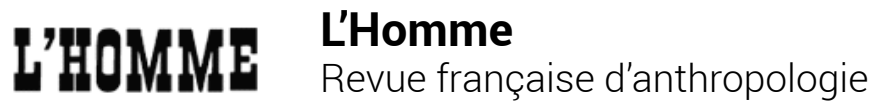

160 | octobre-décembre 2001

Droit, coutume, mémoire

\section{Sjoerd R. Jaarsma \& Marta A. Rohatynskyj, eds, Ethnographic Artifacts. Challenges to a Reflexive Anthropology}

Honolulu, University of Hawai'i Press, 2000, VIII +255 p., réf., index

Francis Affergan

\section{OpenEdition}

\section{Journals}

Édition électronique

URL : http://journals.openedition.org//homme/7926

DOI : 10.4000/lhomme.7926

ISSN : 1953-8103

\section{Éditeur}

Éditions de l'EHESS

Édition imprimée

Date de publication : 1 janvier 2001

Pagination : 276-278

ISBN : 2-7132-1391-6

ISSN : 0439-4216

\section{Référence électronique}

Francis Affergan, «Sjoerd R. Jaarsma \& Marta A. Rohatynskyj, eds, Ethnographic Artifacts. Challenges to a Reflexive Anthropology ", L'Homme [En ligne], 160 | octobre-décembre 2001, mis en ligne le 31 mai 2007, consulté le 24 septembre 2020. URL : http://journals.openedition.org/lhomme/7926 ; DOI :

https://doi.org/10.4000//homme.7926

Ce document a été généré automatiquement le 24 septembre 2020

(C) École des hautes études en sciences sociales 


\title{
Sjoerd R. Jaarsma \& Marta A. Rohatynskyj, eds, Ethnographic Artifacts. Challenges to a Reflexive Anthropology
}

\author{
Honolulu, University of Hawai'i Press, 2000, vIII + 255 p., réf., index
}

Francis Affergan

1 PARMI toutes les aires socioculturelles, l'Océanie apparaît de plus en plus encline, comme objet d'étude et comme sujet de son propre discours, à remettre en question un certain nombre de dogmes qui, au dire de nombreux anthropologues, ont fait les beaux jours de la discipline. Cet ouvrage collectif, dirigé par Sjoerd R. Jaarsma et Marta A. Rohatynskyj, s'efforce de préciser les raisons d'une telle reconfiguration culturelle et d'une telle réinterrogation de l'ethnologie.

2 En premier lieu, l'Océanie hérite d'un passé colonial beaucoup plus massif et douloureux qu'ailleurs. Cet héritage colonial se situe en fait à un double niveau : celui de l'acculturation et celui de l'implication ethnologique. À la différence, par exemple, des Antilles, avec lesquelles de nombreux chercheurs les comparent, les terres océaniennes, déjà occupées par des communautés fortement implantées, ont reçu de plein fouet des cultures exogènes sous diverses formes, dont la moins importante n'est pas celle que revêt l'importation artificielle de valeurs déjà construites. Quant à l'implication ethnologique d'un tel dispositif, il est facile de la mesurer : l'effacement des identités locales déjà échafaudées devant celles contenues dans les préreprésentations et les représentations de l'ethnologie occidentale.

3 En second lieu, cette "crise de la représentation » (p.1) se vérifie dans la mesure où c'est précisément en Océanie (Papouasie-Nouvelle-Guinée, Hawaï...) que les écrits des anthropologues occidentaux se voient, sans doute plus qu'ailleurs pour les raisons invoquées plus haut, commentés, passés au crible de la critique, interprétés par les natifs qui, ce faisant, en s'appropriant des descriptions et des catégories axiologiques, deviennent à leur tour savants. Dans cette perspective, le présent ouvrage se propose 
d'examiner les conditions de «production, distribution et réception de l'ethnographie à la fin de ce siècle en Océanie » (ibid.). La thèse est d'emblée assertée : l'ethnographie est artificielle aussi bien dans les modalités de sa fabrication matérielle que dans les objectifs qu'elle s'assigne.

4 Le premier niveau de cette artificialité se mesure à l'effondrement des liens qui devraient normalement conjoindre l'audience et les sujets de la discipline. Lorsque l'ethnologie prétend représenter les « autres ", elle ne fait rien moins que les incarner et les incorporer dans les pré-conceptions qui la constituent. Le deuxième niveau de cette artificialité s'évalue à l'aune du problème de la réflexivité du chercheur qui ne peut s'absenter ni des données recueillies ni, sur un plan plus épistémologique, des conséquences cognitives du terrain dans la construction même du texte. Le troisième niveau de l'artificialité de l'ethnologie concerne directement le phénomène de la monographie dans son événementialité matérielle comme dans son déploiement scientifique. Les auteurs s'emploient à montrer qu'une étude monographique ne se réduit pas à l'existence d'un simple livre (p.4), mais qu'elle remplit aussi la fonction d'un butoir, d'un vestige historique, d'une espèce d'article de base, première, comme on dit d'une matière.

5 En fabriquant un texte ethnographique, le chercheur élabore donc aussi son objet. C'est John Van Maanen qui aurait le premier introduit cette notion d'« objet fabriqué » (pp. 6-7). Un texte ethnographique ne pourrait plus prétendre refléter des « configurations d'idées » désintéressées, comme s'il possédait cette capacité surnaturelle de s'exempter de sa propre opacité. Il artificialiserait plutôt, en les concrétisant, des collections de relations sociales très complexes (ibid.). Fabriquer ethnographiquement des « domaines d'interprétation textuelle» en les tissant avec la «signification sociale» (p. 8) de la discipline, tel est bien le travail de l'ethnologue qui finit par produire historiquement, parfois à son insu, un certain type de relations entre les cultures de la science ethnologique et celles des colonies ou des ex-colonies.

6 À quelles conditions, se demandent les éditrices de ce collectif, «l'ethnographie d'une communauté est-elle acceptée ou rejetée " par les natifs eux-mêmes? Tel semble être le fil conducteur du questionnement de tout le volume. Devant la globalisation des audiences possibles, il paraît peu contestable, aux yeux des auteurs, que les communautés natives soient en passe d'exiger un contrôle plus légitime de leurs propres représentations. Inversement, et à l'instar de la mécanique des fluides, l'ethnologie se trouve déjà dans une posture où son pouvoir s'amenuise au fur et à mesure que les descriptions et interprétations sont lues et commentées.

7 La réflexivité ethnologique n'est néanmoins pas si démunie qu'elle ne soit à même de poser des problèmes qui, pour cruels qu'ils lui apparaissent, n'en fixent pas moins de nouvelles règles pour la direction de l'esprit : qui construit les identités de ceux dont elle parle ? L'ethnographe, les intéressés ou bien tous ensemble dans un dialogue tissé à l'infini? Ces questions conduisent Sjoerd R. Jaarsma à relativiser considérablement l'autorité dont jouit la discipline, qu'elle fait désormais dépendre plus de l'acceptation ou du refus de son pouvoir que d'une assise scientifique légitimée par le seul travail de la rationalité.

8 Il va sans dire qu'une telle remise en question des canons de l'anthropologie sociale et culturelle n'est pas nouvelle et s'appuie, comme les auteurs eux-mêmes le rappellent (voir notamment Toon Van Meijl, p. 100) sur des travaux fondateurs dans le fil desquels ils se situent. Fredrik Barth, par exemple, avait déjà concentré son objectif sur un 
réexamen du concept de culture qui ne pouvait plus, selon lui, se référer à une notion de partage commun dans une société donnée, mais relevait de plusieurs niveaux : «la multivocalité, la multiplicité des interprétations possibles» et les phénomènes transcientifiques. En d'autres termes, et c'est la thèse que défendra Jonathan Friedman à la fin de l'ouvrage, tous «les concepts sont enchâssés dans des pratiques" sociales. C'est la raison pour laquelle tous les points de vue, quels qu'ils soient, y compris celui de la science ethnologique, proviennent de positions « singulières » et contextualisées. Aussi n'est-il pas étonnant que les collaborateurs de ce volume défendent, en fin de compte, la thèse au terme de laquelle les significations des cultures des autres - les leurs incluses - ressortissent à des procédés constructifs qui rendent contestable le socle sémantique qu'on a cru implanter une fois pour toutes.

À partir de ce noyau dur, tour à tour épistémologiquement déconstruit et reconstruit par Fredrik Barth, une relecture d'un certain nombre de thèmes classiques est entreprise (Niko Besnier, p. 36 ; Toon Van Meijl, p. 88) : les identités ne dépendraient plus d'un ancrage dans une liste de catégories immobiles, mais se négocieraient en permanence en fonction des alliances, mésalliances, affiliations et renversements d'intérêts; le discours scientifique devient dialogique et promeut la question de savoir à qui et de qui, et non plus seulement sur quoi, l'on parle.

10 La collaboration de Jonathan Friedman, incontestablement la plus importante, ne se veut pas seulement conclusive, mais opère une synthèse de surplomb qui lui donne un relief $d$ '«Aufhebung ». Il y défend quatre idées: le terrain est primordialement une relation sociale; l'hybridité, notion qui sature aujourd'hui les analyses relatives à l'anthropologie antillaniste, océaniste et nord-américaniste, ne serait que le cachemisère du retour de l'essentialisme; la globalisation culturelle s'apparente plus au mirage lié aux désirs inavoués des anthropologues postmodernistes qu'à la conséquence rigoureuse d'une analyse scientifique, dans la mesure où elle a toujours été présente sous d'autres formes; enfin, nos propres recherches devront être considérées comme « des parties des mondes que nous étudions» (p. 207), socialement contextualisées et représentées.

11 La recherche ethnologique relève de la pratique sociale et inclut de ce fait des «processus d'interaction et de négociation » (Sjoerd R. Rohatynskyj \& Marta Jaarsma, p.6). Autant il est difficile de répudier un tel constat, autant on est en droit de s'interroger sur les intentions des auteurs. On ne feindra pas un certain trouble après avoir lu le livre. Cet embarras découle de la tension entre certaines thèses défendues ici et apparentées au courant postmoderne, et les idées soutenues par Jonathan Friedman qui semble ne pas vouloir les endosser. Outre son caractère programmatique, force est d'admettre l'aspect flou du projet, tant il est vrai qu'au-delà de la constatation d'un décalage entre les productions scientifiques, ceux sur qui elles portent et les destinataires putatifs, il est pour le moins malaisé d'imaginer concrètement comment les choses se passeraient si l'on devait suivre les recommandations des auteurs. Sauf à récuser toute la démarche ethnologique. Ce qui nécessiterait sans doute une entreprise analytique et critique beaucoup plus affermie. 


\section{AUTEUR}

\section{FRANCIS AFFERGAN}

Université René Descartes-Paris-V. 